\title{
ANÁLISE DE FALHA EM TUBO RETO UTILIZADO EM TROCADOR DE CALOR APLICADO NA INDÚSTRIA SUCROALCOOLEIRA
}

\section{FAILURE ANAL YSIS OF A HEAT-EXCHANGE TUBE APPLIED IN THE SUGAR- ALCOHOL INDUSTRY}

\author{
Otávio Contart Gamboni ${ }^{\mathrm{I}}$ \\ Rosamel Muñoz RiofanoII \\ Gustavo Coser Monteiro Dias ${ }^{\mathrm{III}}$ \\ Edson Luis Boldrin ${ }^{\text {IV }}$ \\ Maurício Angeloni ${ }^{\mathrm{V}}$
}

\begin{abstract}
RESUMO
O trabalho apresenta a análise de falhas de um tubo de trocador de calor aplicado na indústria sucroalcooleira, que falhou em serviço. As amostras foram recebidas após a falha para análises micro e macrográficas, microdureza, análises dimensionais e composição química. Após a realização dos ensaios e análises, constatou-se a formação de grãos ferríticos mais refinados próximos à borda interna do tubo e uma concentração maior de perlita nos contornos de grão. Essa concentração de perlita pode fragilizar localmente o material, podendo indicar uma direção preferencial para a nucleação, propagação de trincas e fraturas no material. Tais fenômenos acontecem devido a degradação microestrutural e o material perde sua resistência mecânica, resultante das condições severas às quais esse componente operava em todo o funcionamento da caldeira a elevada temperatura, fluido corrosivo e não utilização de proteção à corrosão. Mais detalhamentos sobre as condições de funcionamento dos equipamentos podem ser necessários para uma análise mais precisa. No entanto, recomenda-se a mensuração das reais condições de operação, a fim de medir a temperatura, pressão e características do meio a que esses tubos estão submetidos. Também, pode ser aplicado um revestimento térmico protetor para diminuir a taxa de redução de espessura da parede do tubo causada por corrosão, oxidação ou erosão. Realizar ações corretivas como limpeza periódica dos tubos, a fim de minimizar a ocorrência desse tipo de falha no componente analisado.
\end{abstract}

Palavras-chave: Análise de Falha. Aço ASTM A178. Tubos para Caldeira.

\footnotetext{
I Prof. Dr. da Faculdade de Tecnologia Deputado Waldyr Alceu Trigo de Sertãozinho (Fatec-Stz) - São Paulo Brasil. E-mail: otavio.gamboni@fatec.sp.gov.br

II Profa. Dra. da Faculdade de Tecnologia Deputado Waldyr Alceu Trigo de Sertãozinho (Fatec-Stz) - São Paulo Brasil. E-mail: rosamel.riofano@fatec.sp.gov.br

III Prof. Ms. da Faculdade de Tecnologia Deputado Waldyr Alceu Trigo de Sertãozinho (Fatec-Stz) - São Paulo Brasil. E-mail: Gustavo.dias10@fatec.sp.gov.br

${ }^{\text {IV }}$ Currículo sucinto, vinculação corporativa e endereço de contato (e-mail). Ex.: Tecnólogo em Mecânica: Processos de Soldagem - Faculdade de Tecnologia Deputado Waldyr Alceu Trigo de Sertãozinho (Fatec-Stz) - São Paulo Brasil. E-mail: edson.boldrin@fatec.sp.gov.br

v Prof. Dr. da Faculdade de Tecnologia Deputado Waldyr Alceu Trigo de Sertãozinho (Fatec-Stz) - São Paulo Brasil. E-mail: mauricio.angeloni@fatec.sp.gov.br
} 


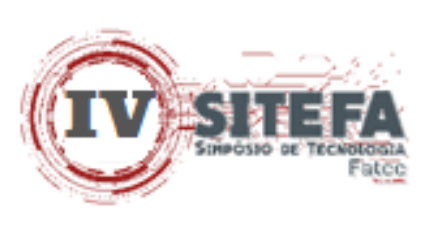

\begin{abstract}
The work presents the failure analysis of a heat-exchange tube applied in the sugar-alcohol industry, which failed in service. The sample was received after failure for micro and micrographic analysis, microhardness test, dimensional analysis and chemical composition verification. After carrying out all these tests and analyses, it was found the formation of refined ferritic grains near the inner edge of the tube and a higher concentration of pearlite in the grain boundaries. This concentration of pearlite can locally embrittle the material and may indicate a preferential direction for nucleation and propagation of cracks and fractures in the material. Such phenomena happened due microstructural degradation and the material loses its mechanical resistance, resulting from the severe conditions under which this component operated throughout the boiler operation at high temperature, corrosive media and non-use of corrosion protection. More details about the equipment's operating conditions may be necessary for a more accurate analysis. However, it is recommended to measure the actual operating conditions, in order to measure the temperature pressure and characteristics of the environment to which these tubes are submitted. Also, a protective thermal coating can be applied to decrease the rate of tube wall thickness reduction caused by corrosion, oxidation, and erosion. Carry out corrective actions such as periodic cleaning of the tubes, in order to minimize the occurrence of this type of failure in the analyzed component.
\end{abstract}

Keywords: Failure analysis. ASTM A178 steel. Boiler tube.

Data de submissão do artigo: 30/06/2021.

Data de aprovação do artigo: 18/08/2021.

DOI: $10.33635 /$ sitefa.v4i1.179

\title{
1 INTRODUÇÃO
}

A falha de um componente em uma indústria é algo completamente indesejável e acarreta diversos custos de maneira direta e indireta. Se esse componente está localizado em uma linha de produção, o prejuízo imediato é a parada da linha para reparo ou substituição do componente. Adicionalmente ao tempo parado para manutenção corretiva, ainda existe o gasto de substituição do componente e ainda, de maneira mais catastrófica, a possibilidade da ocorrência de algum acidente com prejuízos incalculáveis.

Os motivos responsáveis por resultar na falha de um determinado componente podem ser diversos, tais como: escolha equivocada do material, condições ambientais diferentes daquelas solicitadas originalmente quando da fabricação do componente, presença de defeitos de fabricação do componente etc. Ali et al. (2020) apresentou diversos motivos pelos quais alguns trocadores de calor podem falhar, destacando a elevada temperatura e cargas excessivas.

Caldeiras, trocadores de calor e superaquecedores são muito comuns de serem encontrados em diversas indústrias do setor sucroalcooleiro. Esses equipamentos normalmente operam em condições severas de corrosão, altas temperaturas e, em alguns casos, sobre carga mecânica. Assim sendo, os materiais utilizados para confecção desses equipamentos devem possuir algumas características e propriedades para essa finalidade, ou seja, elevada resistência mecânica a quente e resistência à corrosão. Santos et al. (2011) fizeram uma extensa explanação sobre os diversos materiais utilizados para a confecção de caldeiras e turbinas. 


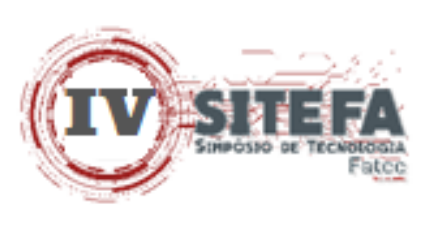

De acordo com Malek et al. (2007), apesar de uma caldeira ser um equipamento relativamente simples em termos de funcionamento, uma vez que produz vapor de água a partir dessa no estado líquido, as atividades de operação e manutenção, se feitas de maneira inadequada poderão acarretar sérios problemas.

Os autores Malek et al. (2007), ainda afirmam que caldeiras são equipamentos de elevada eficiência e que ela pode ser comprometida em casos de ausência de cuidados com a manutenção. Segundo Flynn (2009) a elevada eficiência desse tipo de equipamento dá-se, não exclusivamente, à facilidade de transportar energia de um ponto a outro na forma de vapor d'água. Ainda de acordo com Flynn (2009), vários são os processos nos quais as caldeiras podem ser inseridas, como, por exemplo, na calefação de ambientes (dormitórios, salas comerciais etc.), operações de aquecimento industrial em diversos seguimentos (alimentício, químico, petrolífero, papel e celulose e geração de energia elétrica).

O presente trabalho apresenta a análise de falha realizada para um componente utilizado em caldeiras e superaquecedores de uma indústria do setor sucroalcooleiro. O componente analisado foi um tubo reto, fabricado com o aço ASTM A178 Gr. A, localizado na região da fornalha, revestido por concreto refratário e posicionado frente ao tubulão de lama de uma caldeira aquatubular. A escolha de materiais cuja destinação são componentes que operam em alta pressão deve ser feita de acordo com o código ASME Seção II (MALEK et al., 2007).

\section{MATERIAIS E METODOLOGIA EMPREGADA}

A seguir apresenta-se o componente recebido para estudo, assim como as regiões selecionadas a partir desse para o encaminhamento das análises químico, dimensionais, micrográfica óptica e microdureza das respectivas amostras.

\subsection{Tubo reto fabricado em aço ASTM A178 Grau A}

A Figura 1, apresenta a amostra do tubo reto recebida para análise, com detalhes das extremidades do tubo. De acordo com a indústria em que o tubo estava instalado, o componente ficava localizado acima da região da fornalha, revestido por concreto refratário e posicionado frente ao tubulão de lama de uma caldeira aquatubular modelo AZ-365.

A Tabela 1 apresenta a especificação do tubo relacionada à composição química nominal do aço ASTM A178 Gr. A. 


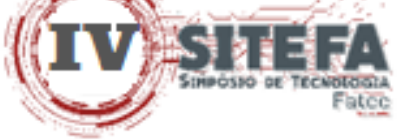

Figura 1- Amostra recebida para análise do tubo reto. Detalhe das extremidades
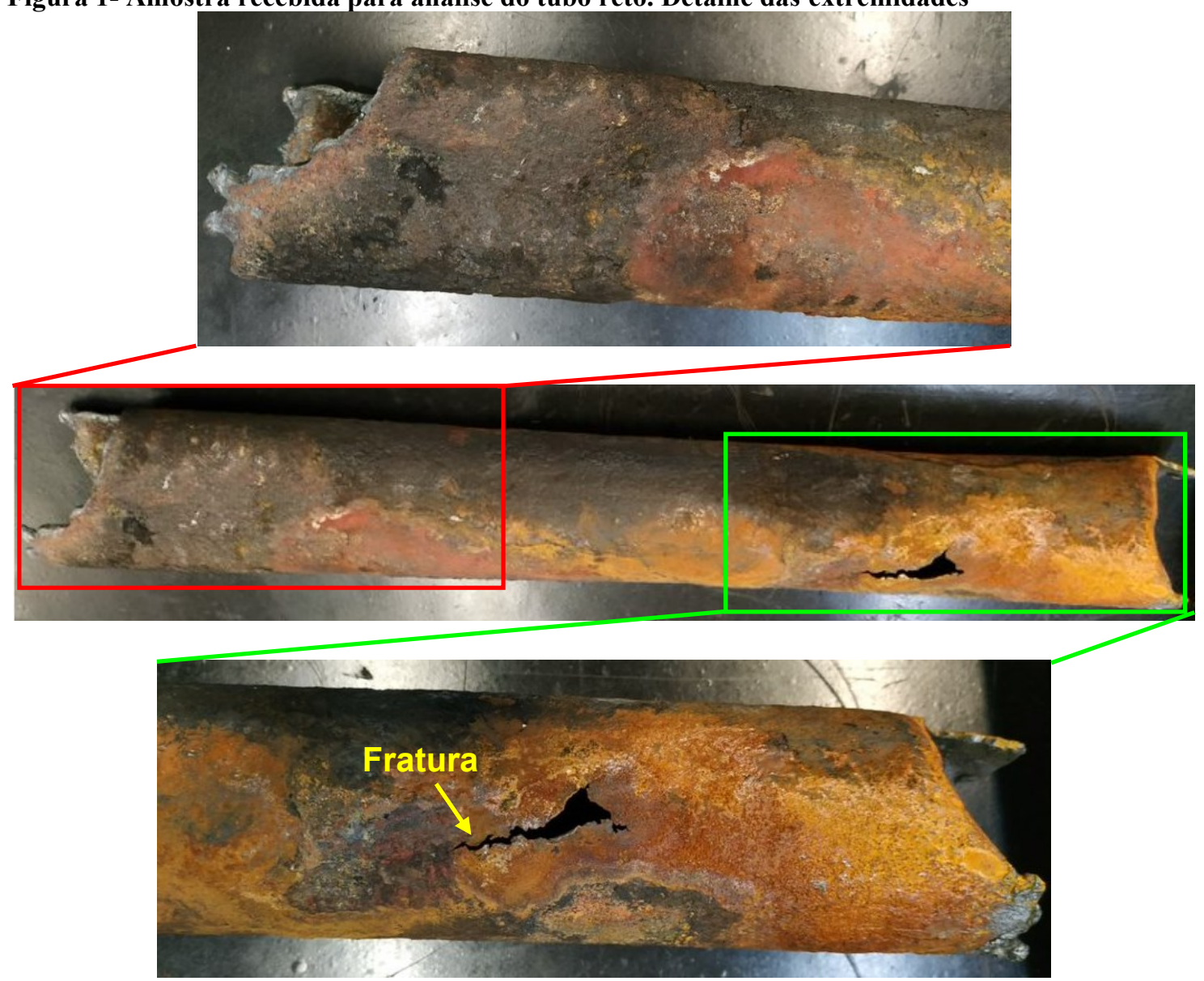

Fonte: próprios autores (2021)

Tabela 1 - Especificação para composição química nominal (\% em peso) do aço

\begin{tabular}{cccccc}
\hline Aço & C & Mn & P & S & Fe \\
\hline ASTM A178 Grau A & $0,06-0,19$ & $0,27-0,63$ & $\begin{array}{c}0,035 \\
\max .\end{array}$ & $\begin{array}{c}0,035 \\
\max .\end{array}$ & Bal. \\
\hline
\end{tabular}

Fonte: Norma ASTM A178/A178M (2019)

\subsection{Análises e ensaios realizados}

A fim de verificar se as composições químicas das amostras estavam de acordo com as especificações descritas pelas normas referentes a cada material, foi feita análise química via espectrometria de emissão óptica por centelhamento. Adicionalmente, análises micro e macroestrutura foram realizadas nas amostras de acordo com as normas ASTM E3-17 e E40715. Foram realizadas 5 medidas de microdureza, para obter a média e o desvio padrão, segundo norma ASMT E384-17.

A Figura 2 apresenta a região onde foram retiradas as amostras para a realização da análise metalográfica. 


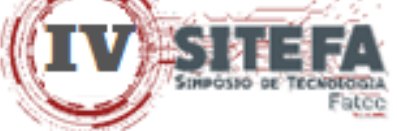

Figura 2 - Região onde foi retirada a amostra para realização da análise metalográfica

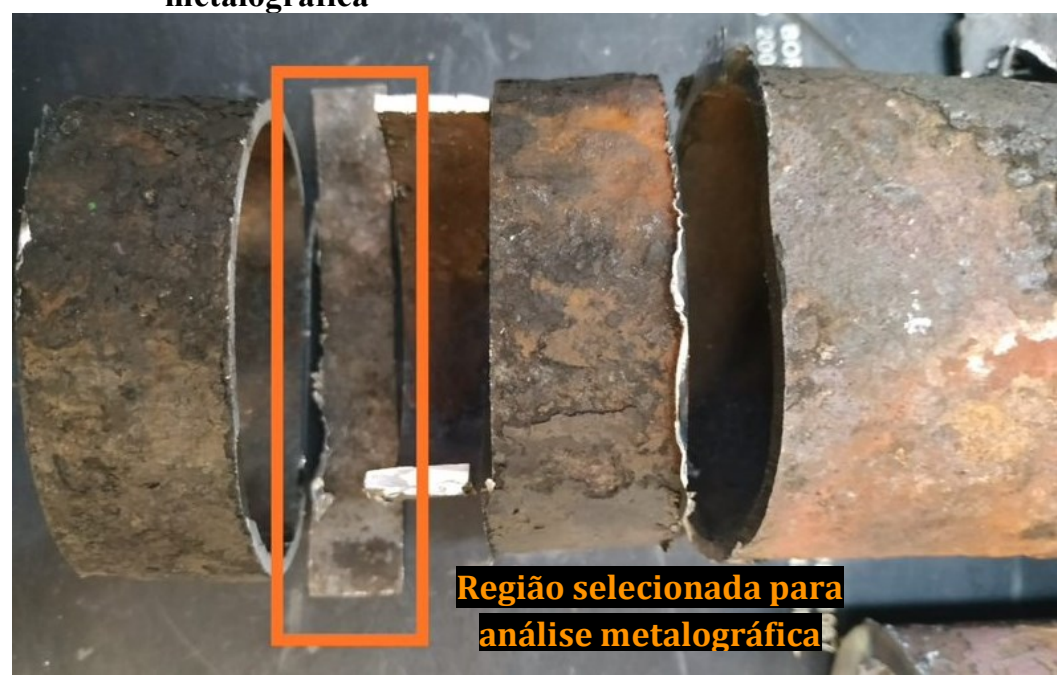

Fonte - próprios autores (2021)

\section{RESULTADOS E DISCUSSÕES}

A Tabela 2 apresenta a composição química do componente analisado. Observa-se que a composição química está dentro dos limites especificados pela norma de referência (Tabela $1)$.

Tabela 2 - Composição química obtida para a amostra recebida

\begin{tabular}{c|c}
\hline Elementos & Amostra ASTM A178 Gr. A (\% em peso) \\
\hline $\mathrm{C}$ & 0,074 \\
\hline $\mathrm{Mn}$ & 0,320 \\
\hline $\mathrm{P}$ & 0,024 \\
\hline $\mathrm{S}$ & 0,022 \\
\hline $\mathrm{Si}$ & 0,012 \\
\hline
\end{tabular}

Fonte: próprios autores (2021)

A Tabela 3 mostra os resultados das medidas de microdureza Vickers (HV), com carga de 9,8N, na superfície do tubo, após a remoção da camada de óxido.

Tabela 3 - Valores de microdureza na superfície do tubo

\begin{tabular}{|c|c|c|c|c|c|c|c|}
\hline \multirow{2}{*}{ Amostra } & \multicolumn{5}{|c|}{ Microdureza $\mathbf{H V}_{9,8 \mathrm{~N}}$} & \multirow{2}{*}{ Média } & \multirow{2}{*}{ DP } \\
\hline & $\mathbf{1}^{\mathrm{a}}$ & $2^{a}$ & $3^{a}$ & $4^{a}$ & $5^{a}$ & & \\
\hline ASTM A178 Gr. A & 94,4 & 95,2 & 94,3 & 95,5 & 94,1 & 94,7 & 0,5 \\
\hline
\end{tabular}

Fonte: próprios autores (2021)

A Tabela 4 apresenta o resultado da análise dimensional do tubo, referente a medição da espessura (da parede) ao longo do componente. 


\section{(iv) $\sin$ EA}

A partir das medições da espessura da parede do tubo, pode-se observar acentuada diminuição com redução da ordem de $27 \%$, quando comparado a espessura original $(3,40 \mathrm{~mm})$, provavelmente causada pelo processo de erosão, corrosão ou oxidação, o que leva ao aumento de tensão.

Tabela 4 - Análise dimensional da espessura (da parede) do tubo

\begin{tabular}{|c|c|}
\hline Amostra & Medidas [mm] \\
\hline \multirow{7}{*}{ Amostra ASTM A178 Gr. A } & 2,40 \\
\hline & 2,90 \\
\hline & 2,85 \\
\hline & 2,95 \\
\hline & 2,00 \\
\hline & 2,10 \\
\hline & 2,10 \\
\hline Média & 2,47 \\
\hline Desvio padrão & $\mathbf{0 , 4 2}$ \\
\hline
\end{tabular}

Fonte: próprios autores (2021)

A Figura 3 apresenta o resultado da análise microestrutural, realizada no tubo, em que é possível observar uma matriz ferrítica com formação de perlita nos contornos de grão. Observa-se uma tendência a formação de grãos ferríticos mais refinados próximos à borda interna do tubo, assim como uma concentração maior de perlita nos contornos de grão. Recomenda-se a realização de ensaio de difração por raio X para aprofundamento. A maior concentração de perlita pode fragilizar localmente o material, podendo indicar uma direção preferencial para a nucleação e propagação de trincas. 


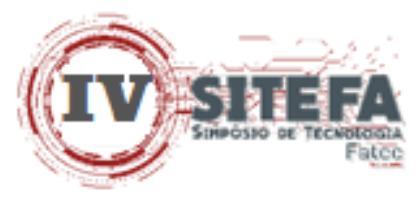

Figura 3 - Micrografia realizada em três regiões distintas do material após ataque com Nital, revelando uma matriz ferrítica com perlita nos contornos de grãos. (a) Borda externa, (b) centro e (c) borda interna. Aumento de 100x e 200x
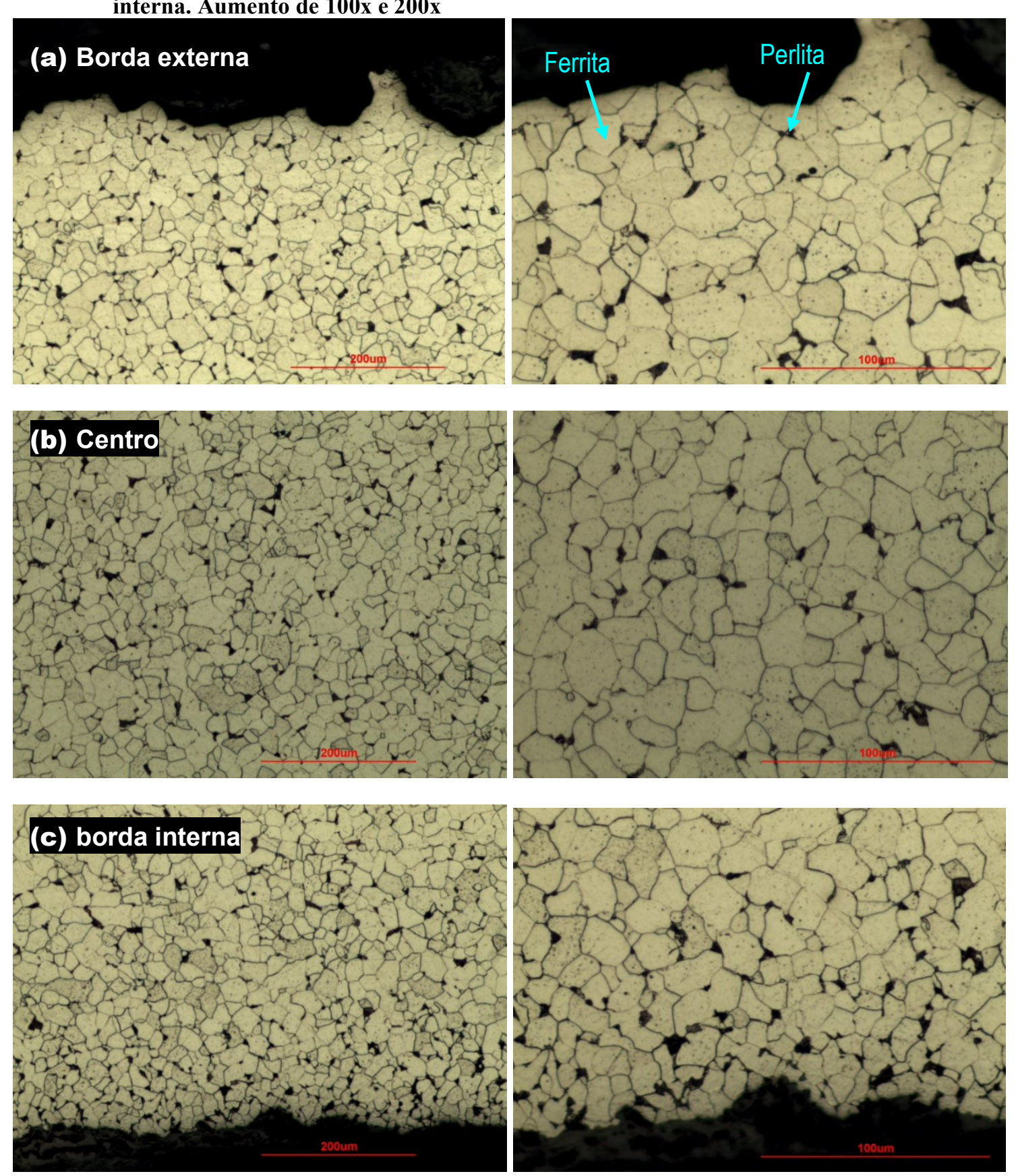

Fonte: próprios autores (2021)

A Figura 4 apresenta as macrografias da região próxima à falha. 


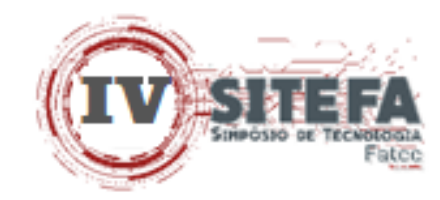

Figura 4 - Macrografias da: (a) amostra do tubo fraturado, (b) mostra uma fratura de aproximadamente 45 mm de comprimento, (c) concentração de óxidos e (d) redução de espessura e deformações plásticas
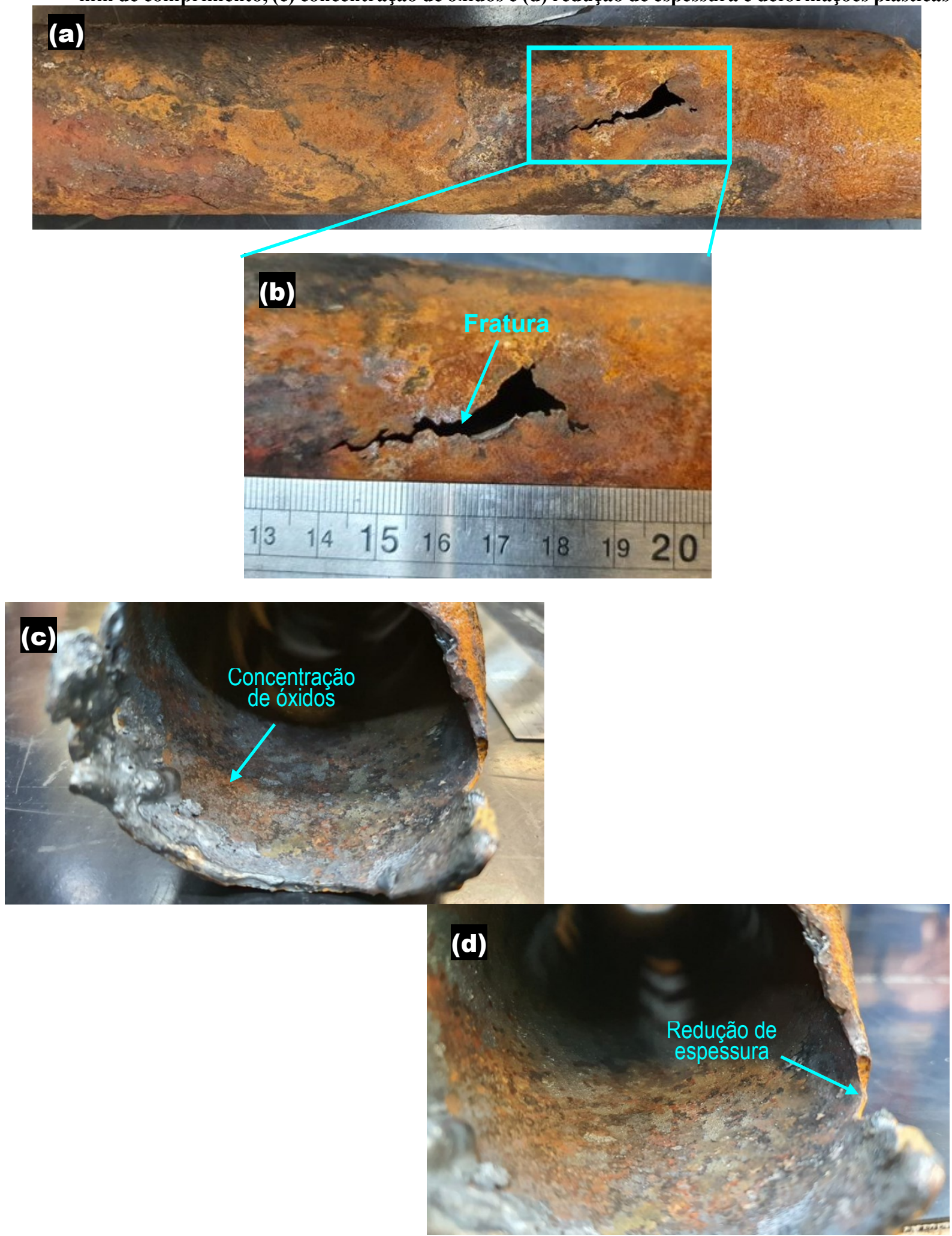

Fonte: próprios autores (2021) 


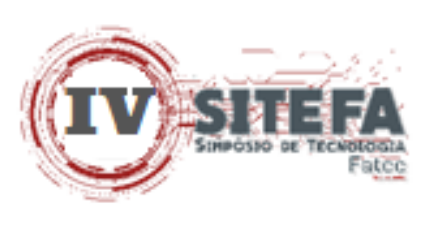

Nota-se a presença de deformação plástica na região destacada em Figura 4(d), próxima ao local em que houve redução de espessura. Essa diminuição de espessura provavelmente deuse pelo processo combinado de oxidação acompanhado do destacamento do metal base com a camada de óxido depositada, uma vez que a região de menor espessura coincide com elevada concentração de óxidos, tanto na superfície externa quanto na interna.

\section{CONCLUSÕES}

A partir dos dados fornecidos pelo solicitante e das análises e ensaios realizados, podese concluir que a fratura ocorreu pela perda de resistência, resultante da degradação microestrutural e oxidação, provocadas pelos carregamentos térmicos, pressão e ao meio em que eles estão expostos. No entanto, ressalta-se que um registro mais detalhado de todas as informações fornecidas pelo solicitante se faz necessário, para o acompanhamento de todas as variáveis do processo, sendo assim de extrema importância para auxiliar na acuracidade das análises das causas de falha, melhorando dessa forma os resultados alcançados.

Apesar de o aço ASTM A178 Gr. A ter suas especificações para operação em paredes d'aguas e feixes tubulares de caldeiras recomenda-se a mensuração das reais condições de operação do mesmo, a fim de medir temperatura, pressão e características do meio em que esses tubos estão submetidos, pois em geral, a degradação desses componentes acaba por resultar em alterações em sua microestrutura, o que resulta em diminuição da resistência. Ou seja, as mudanças microestruturais são indicativos da perda de resistência mecânica no material.

As regiões localmente degradadas do material terão uma dureza mais baixa, como comprovado pelas análises. Essas medidas podem fornecer indicações suplementares úteis do estado de danos dos materiais estudados.

Sugere-se ações corretivas, que podem incluir a determinação da vida remanescente, baseada na temperatura efetiva, nível de tensão e/ou características do material, por meio de métodos de análise paramétricos aplicados para avaliar os efeitos das várias ações corretivas, tais como o modelo de Monkman - Grant; Larson-Miller; Manson-Haferd; Orr-Sherby-Dorn; Manson-Brown; Goldhoff-Sherby; White-Le May.

Flushing com fluido sob alta pressão, ou limpeza química podem ser realizados para remover incrustações internas, deposições, entupimentos e reduzir a temperatura do metal do tubo. Elevação do grau dos materiais podem ser necessárias para tornar os respectivos aços mais resistentes nos locais de alta temperatura.

Ações corretivas tais como telhas de proteção, aplicação de revestimento térmico protetor pode ser feita para reduzir a taxa de redução de espessura da parede do tubo causada por corrosão, oxidação ou erosão.

\section{REFERÊNCIAS}

ALI, M.; Ul-Hamid, A.; Alhems, L. M.; Saeed, A. Review of common failures in heat exchangers - Part I: Mechanical and elevated temperature failures. Engineering Failure Analysis, v. 109, 2020.

AMERICAN SOCIETY FOR TESTING AND MATERIALS (ASTM). ASTM

A178/A178M-19 Standard Specification for Electric-Resistance-Welded Carbon Ateel and Carbon-Manganese Steel Boiler and Superheater Tubes, 2019. 


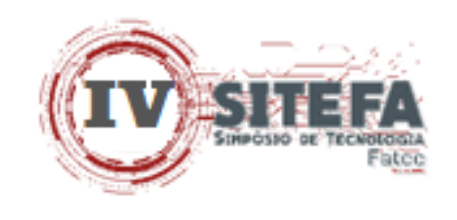

ASTM E3-17 Standard Guide for Preparation of Metallographic Specimens, 2017 . ASTM E407-15 Standard Practice for Microetching Metals and Alloys, 2015

2017 . ASTM E384-17 Standard Test Methods for Microindentation Hardness of Materials,

FLYNN, Daniel. Nalco water handbook. McGraw-Hill Education, 2009.

MALEK, Mohammad A. et al. Heating Boiler Operator's Manual: Maintenance, Operation, and Repair. McGraw-Hill Education, 2007. ASME seção II

SANTOS, D.; Cabrita, I.; Gulyurtlu, I. Materiais para caldeiras e turbinas de centrais termoeléctricas avançadas. Ciência e Tecnologia dos Materiais. v. 23. n. 1/2, 2011. 